\title{
Characterizing Effortful Swallows from Healthy Community Dwelling Adults Across the Lifespan Using High-Resolution Cervical Auscultation Signals and MBSImP Scores: A Preliminary Study
}

\author{
Cara Donohue $^{1}$ (D) Yassin Khalifa ${ }^{2} \cdot$ Subashan Perera ${ }^{3} \cdot$ Ervin Sejdić $^{2,4,6} \cdot$ James L. Coyle ${ }^{1,5}$
}

Received: 10 November 2020 / Accepted: 10 September 2021 / Published online: 18 September 2021

(c) The Author(s), under exclusive licence to Springer Science+Business Media, LLC, part of Springer Nature 2021

\begin{abstract}
There is growing enthusiasm to develop inexpensive, non-invasive, and portable methods that accurately assess swallowing and provide biofeedback during dysphagia treatment. High-resolution cervical auscultation (HRCA), which uses acoustic and vibratory signals from non-invasive sensors attached to the anterior laryngeal framework during swallowing, is a novel method for quantifying swallowing physiology via advanced signal processing and machine learning techniques. HRCA has demonstrated potential as a dysphagia screening method and diagnostic adjunct to VFSSs by determining swallowing safety, annotating swallow kinematic events, and classifying swallows between healthy participants and patients with a high degree of accuracy. However, its feasibility as a non-invasive biofeedback system has not been explored. This study investigated 1. Whether HRCA can accurately differentiate between non-effortful and effortful swallows; 2 . Whether differences exist in Modified Barium Swallow Impairment Profile (MBSImP) scores (\#9, \#11, \#14) between non-effortful and effortful swallows. We hypothesized that HRCA would accurately classify non-effortful and effortful swallows and that differences in MBSImP scores would exist between the types of swallows. We analyzed 247 thin liquid $3 \mathrm{~mL}$ command swallows (71 effortful) to minimize variation from 36 healthy adults who underwent standardized VFSSs with concurrent HRCA. Results revealed differences $(p<0.05)$ in 9 HRCA signal features between non-effortful and effortful swallows. Using HRCA signal features as input, decision trees classified swallows with $76 \%$ accuracy, $76 \%$ sensitivity, and $77 \%$ specificity. There were no differences in MBSImP component scores between non-effortful and effortful swallows. While preliminary in nature, this study demonstrates the feasibility/promise of HRCA as a biofeedback method for dysphagia treatment.
\end{abstract}

Keywords Dysphagia · Videofluoroscopy $\cdot$ Machine learning $\cdot$ Cervical auscultation · Biofeedback $\cdot$ Treatment · Deglutition · Deglutition disorders

James L. Coyle

jcoyle@pitt.edu

Cara Donohue

cad191@pitt.edu

1 Department of Communication Science and Disorders, School of Health and Rehabilitation Sciences, University of Pittsburgh, 6035 Forbes Tower, Pittsburgh, PA 15260, USA

2 Department of Electrical and Computer Engineering, Swanson School of Engineering, University of Pittsburgh, Pittsburgh, PA 15260, USA

3 Division of Geriatrics, Department of Medicine, University of Pittsburgh, Pittsburgh, PA 15261, USA
4 Department of Bioengineering, Swanson School of Engineering, University of Pittsburgh, Pittsburgh, PA 15260, USA

5 Department of Otolaryngology, School of Medicine, University of Pittsburgh Medical Center, Pittsburgh, PA 15260, USA

6 Department of Biomedical Informatics, School of Medicine Intelligent Systems Program, School of Computing and Information, University of Pittsburgh, Pittsburgh, PA 15260 , USA 


\section{Introduction}

Within clinical settings, a common challenge for dysphagia practitioners remains the lack of inexpensive, portable, and non-invasive dysphagia management methods available for assessment and treatment. To diagnose dysphagia, instrumental methods remain the gold standard [e.g., videofluoroscopy (VF), fiberoptic endoscopic evaluation of swallowing (FEES)]. While these methods are objective and provide insight into swallowing physiology, there are limitations to performing them including the cost, limited access in some settings (and countries), exposure to radiation (i.e., VF), and inability for some patients to participate in the examination (e.g., patient size, COVID-19 restrictions, patient desire to forgo further imaging studies).

In addition to this, few accurate and non-invasive methods to provide biofeedback during dysphagia treatment are readily available within clinical settings and few clinicians are trained in deploying these methods [1]. FEES and VF have been implemented as biofeedback methods for dysphagia treatment and have been shown to be advantageous for patient/caregiver education and developing individualized treatment plans [2-4]. In fact, clinician feedback and participant/patient performance have been shown to be more accurate for certain swallowing maneuvers using VF compared to other biofeedback methods [e.g., surface electromyography (sEMG)] [3, 4]. However, dysphagia treatment using only VF for biofeedback is unrealistic within clinical settings due to the cost, radiation exposure, and time constraints/accessibility [3, 4]. Due to the limitations of FEES and VF as biofeedback methods for treatment, other non-invasive modalities such as sEMG have been explored. Yet a study examining concurrent VF and sEMG found very weak to moderate correlations between submental sEMG durations and temporal kinematic measures of hyolaryngeal displacement using VF images when participants performed the Mendelsohn maneuver [5]. A recent systematic review that examined biofeedback methods used in dysphagia treatment found that accelerometry, sEMG, and tongue manometry were the most frequently used in research studies [6]. In three studies, visual biofeedback using sEMG and accelerometry led to significantly improved hyoid bone displacement (compared to a control) during dysphagia treatment that targeted functional swallowing exercises such as the effortful swallow and Mendelsohn maneuver [6]. While these results are promising, study limitations included small sample sizes, the heterogeneity of patients, and mixed evidence regarding whether biofeedback results in clinically meaningful, functional changes in swallowing [5-9]. More specifically in studies using accelerometry, low quality studies have been implemented with flawed study designs and the use of subjective and non-validated swallowing outcome measures [6-9].

Due to the limitations of current biofeedback modalities, innovative methods for providing continuous monitoring and biofeedback during dysphagia treatment are under investigation. One such modality is a novel wearable electromyography sensor-array patch that has demonstrated similar signal quality as traditional, commercially available sEMG during water swallow tasks [10]. Another potential biofeedback modality currently being explored is high-resolution cervical auscultation (HRCA) [11]. HRCA is a method of characterizing swallow function that integrates information from acoustic and vibratory signals from non-invasive sensors (contact microphone, tri-axial accelerometer) attached to the anterior laryngeal framework during swallowing. Following collection of HRCA signals, HRCA signal features are extracted using advanced signal processing techniques to use the HRCA signal features as input to machine learning algorithms to provide insight into swallowing physiology using human ratings of VF images as the "ground truth." HRCA has demonstrated promise as a dysphagia screening method and potential diagnostic adjunct to VF by classifying safe and unsafe swallows (as measured by the penetration-aspiration scale) [11-17], tracking hyoid bone displacement in healthy adults and patients with suspected dysphagia [18, 19], annotating temporal swallow kinematic events in healthy adults and patients with suspected dysphagia (e.g., durations of upper esophageal sphincter opening and laryngeal vestibule closure) [20-22], categorizing swallows between healthy participants and different patient populations [23, 24], and detecting clinical ratings of swallow physiology in patients with suspected dysphagia using the Modified Barium Swallow Impairment Profile (MBSImP) [25] with a high degree of accuracy [19, 21]. However, the utility of HRCA's capabilities to noninvasively characterize these physiologic events, many of which are targets of behavioral augmentation via compensatory swallowing maneuvers (e.g., effortful swallow, Mendelsohn maneuver), and differentiate between swallows in which they are accurately deployed without imaging verification, has yet to be investigated. In our clinical work, we have observed difficulty by patients in generalizing training in these maneuvers to accurate performance when assessed using VF, likely due to the lack of ongoing performance evidence in the training stage in which mass practice is deployed in clinic and home programs. Success of such an effort to provide ongoing, noninvasive indications of accurate or inaccurate performance would be of value in demonstrating preliminary efficacy of HRCA as a potential biofeedback method for dysphagia treatment.

Compensatory swallowing maneuvers (e.g., effortful swallow, Mendelsohn maneuver) are common dysphagia rehabilitation techniques that are used to improve swallow 
function in patients with dysphagia by altering swallowing physiology. The effortful swallow is one type of compensatory swallowing maneuver that is frequently deployed in clinical settings for patients with dysphagia and has been explored in research studies in both healthy adults and patients with dysphagia. Following dysphagia treatment targeting the effortful swallow, some patients with dysphagia have exhibited decreased pharyngeal residue and decreased penetration/aspiration, but no changes in upper esophageal sphincter (UES) opening diameter, duration of UES opening, laryngeal elevation, or hyoid movement [26-29]. In healthy adults, the research evidence is mixed regarding the impact of effortful swallows on swallowing physiology. For example, one study in healthy adults found that effortful swallows led to longer durations for temporal swallow kinematic measurements (e.g., hyoid movement duration, duration of UES opening) and increased pyriform sinus residue [30]. Other studies in healthy adults have found no differences in airway protection or swallowing efficiency between non-effortful and effortful swallows [29]. While research studies have examined differences in temporal kinematic measurements between non-effortful and effortful swallows in healthy adults, no studies have examined differences between non-effortful and effortful swallows using a clinical rating tool (e.g., MBSImP), and few researchers have investigated non-invasive or non-imaging alternatives to VF that are capable of determining whether the effortful swallow maneuver is accurately performed once a patient has been properly trained. Such a system holds potential for enhancing clinician judgment of accurate performance (e.g., clinician feedback to patient) which is the source of accurate clinical cuing and patient performance for effortful swallows to mitigate maladaptive learning of the maneuver [3, 4]. Therefore, this study investigated 1 . Whether HRCA can differentiate between non-effortful and effortful swallows performed by the same individuals; 2 . Whether there are differences in MBSImP components \#9 (anterior hyoid excursion), \#11 (laryngeal vestibular closure), and \#14 (pharyngoesophageal segment opening) between non-effortful and effortful swallows. We hypothesized that HRCA combined with signal processing and machine learning algorithms would classify swallows as non-effortful or effortful with a high degree of accuracy and that there would be differences in MBSImP component scores \#9, \#11, and \#14 between non-effortful and effortful swallows.

\section{Methods}

\section{Equipment and Procedures}

The Institutional Review Board for this institution approved this research study. All participants provided written informed consent. Data analyses were performed on 247 thin liquid swallows from 36 healthy community dwelling adults across the lifespan (19 male) between the ages of 49 and 86 (mean age 65.53 \pm 7.67 years). This subset of data is part of an ongoing prospective study that aims to analyze swallow function in healthy community dwelling adults across the lifespan. Participants were enrolled in this research study based on the following inclusionary criteria based on participant report: no history of swallowing difficulties, history of a neurological disorder, prior surgery to the head or neck region, or chance of being pregnant (female participants). Participants underwent a standardized videofluoroscopic swallow study (VFSS) procedure with concurrent HRCA and were imaged in the lateral plane. For non-effortful swallows, participants swallowed ten thin liquid boluses in a randomized presentation order (five $3 \mathrm{~mL}$ boluses via spoon, five self-selected "comfortable" cup sips). For the $3 \mathrm{~mL}$ boluses via spoon, participants were instructed to "Hold the liquid in your mouth and wait until I tell you to swallow it." For the comfortable cup sips, participants were instructed to "Take a comfortable sip of liquid and swallow it whenever you're ready." For effortful swallows, participants swallowed one practice thin liquid water bolus and two $3 \mathrm{~mL}$ thin liquid barium boluses via spoon. During the practice effortful swallow, participants were instructed to "Swallow hard using all your throat muscles." For the effortful swallows that were recorded using VFSSs, participants were instructed to "Hold the liquid in your mouth and wait until I tell you to swallow it" and then to "Swallow hard" during the exam. For analyses purposes, only the $3 \mathrm{~mL}$ thin liquid boluses via spoon were used to compare the non-effortful and effortful swallows to minimize variation (e.g., bolus volume, utensil, command swallow). See Table 1 for the bolus characteristics for swallows used for analyses for this study. The average fluoro time for participants was $1.06 \mathrm{~min}$.

VFSS procedures were conducted using a standard fluoroscopy system (Precision 500D system, GE Healthcare, LLC, Waukesha, WI) at a pulse rate of 30 pulses per second (PPS). A frame grabber module (AccuStream Express HD, Foresight Imaging, Chelmsford, MA) captured the raw video signals at a rate of 73 frames per second (FPS). Prior to analysis, the video files were downsampled to 30FPS. HRCA signals were collected concurrently during the VFSSs via a

Table 1 Bolus characteristics for swallows from the healthy community dweller participants

\begin{tabular}{lll}
\hline Bolus viscosity, utensil, type of swallow & $\begin{array}{l}\text { Number of } \\
\text { swallows }\end{array}$ & $\begin{array}{l}\text { Percentage } \\
\text { of swallows }\end{array}$ \\
\hline $3 \mathrm{~mL}$ thin by spoon non-effortful swallows & 176 & 71.26 \\
$3 \mathrm{~mL}$ thin by spoon effortful swallows & 71 & 28.74 \\
\hline
\end{tabular}

All boluses were administered by spoon with a command to swallow 
tri-axial accelerometer (ADXL 327, Analog Devices, Norwood, Massachusetts) that was powered by a $3 \mathrm{~V}$ output (model 1504, BK Precision, Yorba Linda, California) and a contact microphone. The accelerometer and contact microphone were placed in custom casings to ensure adequate contact for signal acquisition during data collection. The non-invasive HRCA sensors were placed on the anterior laryngeal framework at the level of the cricoid cartilage with tape after cleaning participants' neck region with alcohol pads. The sensors were carefully placed to avoid interfering with VFSS images, to ensure adequate signal acquisition, and to ascertain alignment of the tri-axial accelerometer with the participant's neck $[11,31]$. The precise placement of the accelerometer and contact microphone can be viewed in Fig. 1.

Signals from the accelerometer and the microphone were hardware bandpass filtered from 0.1 to $3000 \mathrm{~Hz}$ (model P55, Grass Technologies, Warwick, Rhode Island), amplified, and digitized using a data acquisition device (National Instruments $6210 \mathrm{DAQ})$ at a sampling rate of $20 \mathrm{kHz}$ with the Signal Express program in LabView (National Instruments, Austin, Texas). Before analysis, the signals were then downsampled to $4 \mathrm{kHz}$ to smooth out high frequency noise.

Prior to data analysis for this study, one trained rater segmented video files into individual swallow segments with ongoing intra-rater reliability within a 3-frame tolerance of $100 \%$ based on randomly re-coding one out of every ten swallows. Another trained rater coded $10 \%$ of swallows for inter-rater reliability with intra-class coefficients (ICCs) of at least 0.9 [32]. The methods for swallow segmentation have been described in previous publications [14, 33]. No other temporal kinematic measurements were performed aside from identifying the onset and offset of each swallow, and the sole purpose of these measurements was to segment the video files into individual swallows.

\section{MBSImP Ratings}

An MBSImP-certified clinician completed all MBSImP ratings for components \#9, \#11, and \#14. Before performing swallowing ratings, inter-rater reliability was established by completing the MBSImP reliability test with at least $80 \%$ exact agreement for all MBSImP component scores. Ongoing intra-rater reliability was maintained by randomly selecting one swallow to re-code every ten swallows with $100 \%$ exact agreement. Inter-rater reliability was conducted on $10 \%$ of swallows by another certified MBSImP clinician with 79\% exact agreement for components \#9, \#11, and \#14.

\section{Pre-Processing and Feature Extraction from HRCA Signals}

An autoregressive model was used to build a digital finite impulse filter to remove the device noise associated with each of the sensors. The filters were designed to remove the baseline noise present in the sensors' output when no physical input is applied. Afterward, motion artifacts and low frequency noise, such as head movement, were removed using fourth-order splines. Finally, wavelet denoising was used to eliminate any additional noise that might exist in the signals

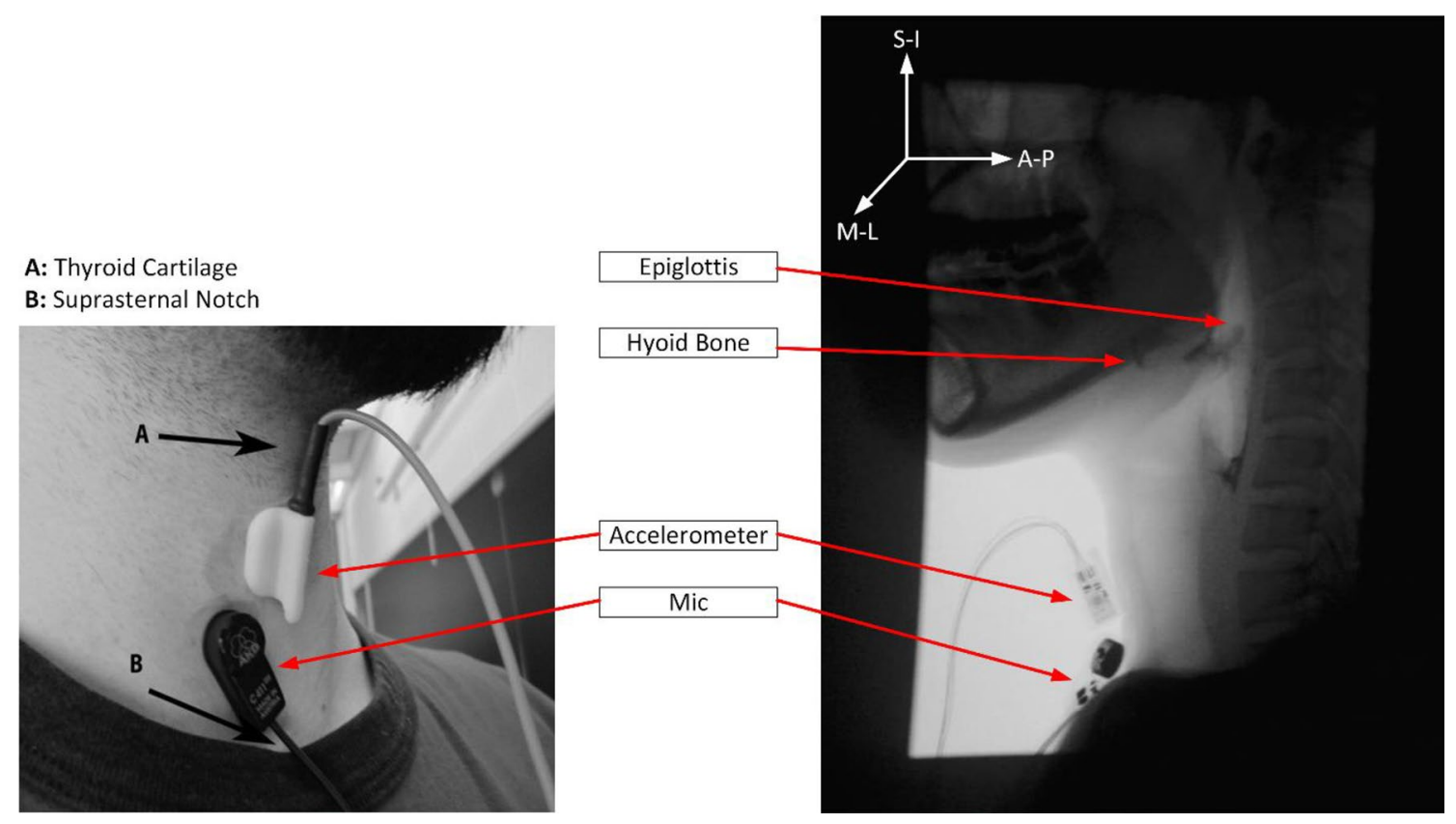

Fig. 1 Placement of HRCA neck sensors during data collection [21] 
$[17,20,21]$. The onset and offset of swallows were taken from the segmented videos after applying the proper sampling mapping between videos and signals. The signals were then segmented using the mapped onset and offset times for feature extraction [33]. A summary of the features extracted from the HRCA signals and the explanations of their meanings can be viewed in Table 2. Nine features were extracted from the contact microphone and the three directions of the tri-axial accelerometer (anterior-posterior, superior-inferior, medial-lateral) for a total of 36 signal features. This set of features has been proven effective in differentiating between HRCA signals from different types of swallows and extraction of multiple swallow kinematics [17, 21, 24, 34].

\section{Data Analysis}

We fit linear mixed models to examine the association between HRCA signal features, non-effortful swallows, and effortful swallows. We used multiple supervised machine learning classifiers (e.g., support vector machines [SVM], Naïve Bayes, decision trees, linear discriminant analysis) that use HRCA signal features as input to classify swallows as non-effortful or effortful. The supervised machine learning classifiers were deployed using the entire set of HRCA signal features $(n=36)$, the features that were statistically significant $(n=9)$, and the linearly independent features [as determined by performing a principal component analysis (PCA)]. A leave-one-out procedure was used to evaluate the classification accuracy of all the used classifiers. A leaveone-out procedure involves training the classifier on the entire data set except for one randomly selected swallow which is used to test the accuracy of the classifier. This training and testing procedure were repeated until all swallows in the data set were tested at least once. The accuracy, sensitivity, and specificity of all supervised machine learning classifiers were then calculated. We fit linear mixed models to determine if there were differences in MBSImP component scores \#9, \#11, and \#14 between the non-effortful and the effortful swallows. SPSS (IBM, Armonk, NY) was used to fit the linear mixed models. MATLAB (The MathWorks, Inc., Natick, MA) and R (The R Foundation) were used to construct and evaluate the performance of the supervised machine learning classifiers.

\section{Results}

Results revealed that there was a statistically significant $(p<0.05)$ difference in 9 HRCA signal features between the non-effortful and effortful swallows. Complete results from the linear mixed model can be viewed in Table 3. From the microphone signals, statistically significant features included

Table 2 Explanation of features extracted from the HRCA signals

\begin{tabular}{lll}
\hline Domain & Signal feature & Significance \\
\hline Time & Standard deviation & Reflects the signal variance around its mean value \\
& Skewness & Describes the asymmetry of amplitude distribution around mean \\
& Kurtosis & Describes the peakness of the distribution relative to normal distribution \\
Information-theoretic & Lempel-Ziv Complexity & Describes the randomness of the signal \\
Frequency & Entropy rate & Evaluates the degree of regularity of the signal distribution \\
& Peak frequency $(\mathrm{Hz})$ & Describes the frequency of maximum power \\
Spectral centroid $(\mathrm{Hz})$ & Evaluates the median of the spectrum of the signal \\
Time-frequency & Bandwidth $(\mathrm{Hz})$ & Describes the range of frequencies of the signal \\
& Wavelet entropy & Evaluates disorderly behavior for non-stationary signal
\end{tabular}

Table 3 Summary of the statistically significant HRCA signal features associated with differentiating between non-effortful and effortful swallows from healthy community dwelling adults across the lifespan

\begin{tabular}{|c|c|c|c|c|c|c|c|c|c|}
\hline & $\begin{array}{l}\text { Standard } \\
\text { deviation }\end{array}$ & Skewness & Kurtosis & $\begin{array}{l}\text { Lempel-Ziv } \\
\text { complexity }\end{array}$ & Entropy rate & $\begin{array}{l}\text { Peak fre- } \\
\text { quency }\end{array}$ & $\begin{array}{l}\text { Spectral cen- } \\
\text { troid }\end{array}$ & Bandwidth & $\begin{array}{l}\text { Wavelet } \\
\text { entropy }\end{array}$ \\
\hline Microphone & $0.0177 *$ & 0.4246 & 0.0936 & 0.1989 & 0.4892 & $0.0330 *$ & $0.0004 *$ & $0.0014 *$ & $0.0161^{*}$ \\
\hline $\begin{array}{l}\text { Anterior-pos- } \\
\text { terior }\end{array}$ & $0.0056^{*}$ & 0.6068 & 0.6068 & 0.3430 & 0.4603 & 0.5481 & 0.7029 & 0.5582 & 0.1718 \\
\hline $\begin{array}{l}\text { Superior-infe- } \\
\text { rior }\end{array}$ & $0.0043^{*}$ & 0.0942 & 0.3582 & 0.9820 & 0.2410 & 0.1750 & 0.2033 & 0.1233 & $0.0443 *$ \\
\hline Medial-lateral & $0.0238^{*}$ & 0.3065 & 0.2134 & 0.5180 & 0.2958 & 0.9400 & 0.5739 & 0.5182 & 0.1152 \\
\hline
\end{tabular}

${ }^{*} p<0.05$ 


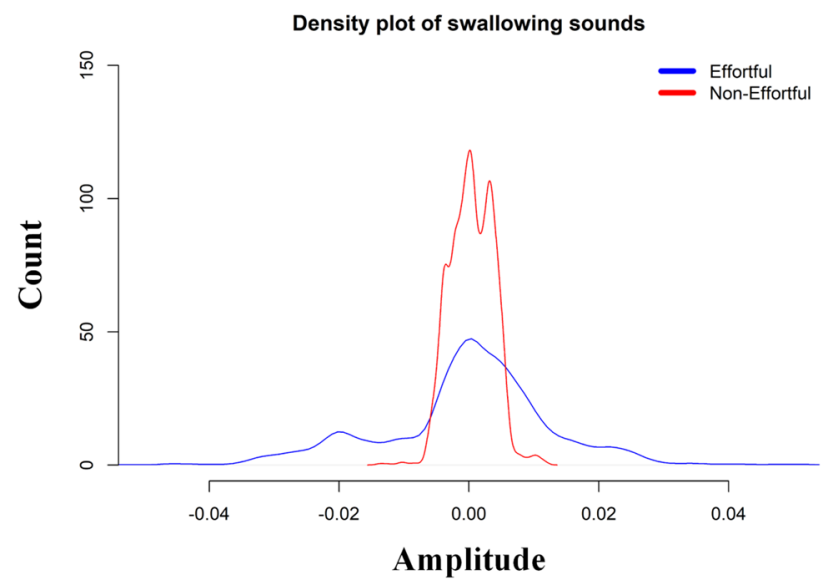

Fig. 2 Density plot from the HRCA microphone signals showing the difference in standard deviation between the non-effortful and effortful swallows

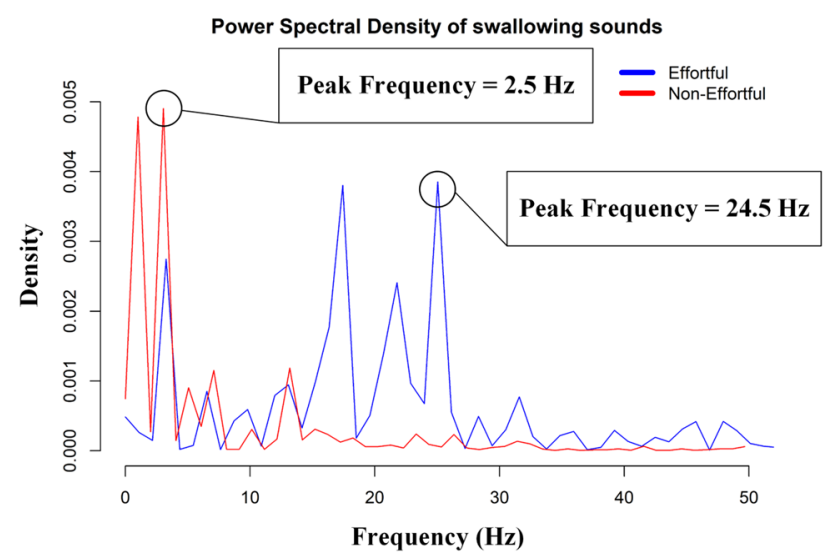

Fig. 3 Power spectral density plot from the HRCA microphone signals showing the difference in peak frequency between the non-effortful and effortful swallows

standard deviation, peak frequency, spectral centroid, bandwidth, and wavelet entropy. From the anterior-posterior and medial-lateral accelerometer axes, standard deviation was the only statistically significant feature. From the superior-inferior accelerometer axis, statistically significant features included standard deviation and wavelet entropy. Figures 2 and 3 illustrate two examples of the differences in signal features (e.g., standard deviation, peak frequency) between the non-effortful and effortful swallows.

After evaluating the performance of all supervised machine learning classifiers using the entire set of HRCA signal features (36), the features that were statistically significant (9), and the statistically independent features; decision trees and linear discriminant analysis had the best performance. Using the 9 most significant HRCA signal features as input, decision trees classified swallows as non-effortful or effortful with $76 \%$ accuracy, $76 \%$ sensitivity, and $77 \%$ specificity. A complete summary of the performance of the different supervised machine learning classifiers can be viewed in Table 4. For MBSImP component scores, results from the linear mixed model revealed that there were no significant differences $(p>0.05)$ in MBSImP component scores \#9, \#11, and \#14 between the non-effortful and effortful swallows. Table 5 shows a complete summary of the MBSImP component scores for the non-effortful and effortful swallows.

\section{Discussion}

This study found that HRCA combined with advanced signal processing and machine learning techniques could accurately and autonomously classify swallows from healthy adults as non-effortful or effortful without imaging. This is of particular clinical interest given the results indicating that analysis of the VF data, which is commonly used to confirm treatment effect in training of the effortful swallow, did not generate significant differences in the MBSImP components measured. While preliminary in nature, these results provide evidence regarding the potential of HRCA as a biofeedback method and an indicator of accurate performance for use by the clinician in providing reinforcement to the patient, for dysphagia treatment protocols in the future. These results

Table 4 Performance of classifiers used to differentiate between non-effortful and effortful swallows from healthy community dwelling adults across the lifespan

\begin{tabular}{|c|c|c|c|c|c|c|c|c|c|}
\hline \multirow[t]{2}{*}{ Classifier } & \multicolumn{3}{|c|}{ Entire set of features ( 36 features) } & \multicolumn{3}{|c|}{$\begin{array}{l}\text { Subset of features ( } 9 \text { most significant } \\
\text { features-Table } 4 \text { ) }\end{array}$} & \multicolumn{3}{|c|}{ Feature selection (PCA) } \\
\hline & Accuracy & Sensitivity & Specificity & Accuracy & Sensitivity & Specificity & Accuracy & Sensitivity & Specificity \\
\hline SVM & 0.575 & 0.69 & 0.547 & 0.541 & 0.539 & 0.544 & 0.671 & 0.647 & 0.705 \\
\hline Naïve Bayes & 0.603 & 0581 & 0.642 & 0.656 & 0.614 & 0.756 & 0.647 & 0.627 & 0.667 \\
\hline Decision Trees & 0.74 & 0.747 & 0.733 & 0.767 & 0.76 & 0.775 & 0.603 & 0.606 & 0.6 \\
\hline $\begin{array}{l}\text { Linear discrimi- } \\
\text { nant analysis }\end{array}$ & 0.562 & 0.557 & 0.567 & 0.61 & 0.589 & 0.643 & 0.616 & 0.605 & 0.631 \\
\hline
\end{tabular}


Table 5 MBSImP ratings for components \#9, \#11, and \#14 for non-effortful and effortful swallows from healthy community dwelling adults

\begin{tabular}{|c|c|c|c|}
\hline MBSImP component & MBSImP score & $\begin{array}{l}\text { Number }(\%) \text { of } \\
\text { non-effortful swal- } \\
\text { lows }\end{array}$ & $\begin{array}{l}\text { Number }(\%) \text { of } \\
\text { effortful swal- } \\
\text { lows }\end{array}$ \\
\hline \multirow[t]{3}{*}{ \#9 Anterior hyoid excursion } & Complete anterior movement $(0)$ & $123(69.89)$ & $49(69.01)$ \\
\hline & Partial anterior movement (1) & $53(30.11)$ & $22(30.99)$ \\
\hline & No anterior movement (2) & $0(0)$ & $0(0)$ \\
\hline \multirow[t]{3}{*}{ \#11 Laryngeal vestibular closure } & Complete; no air/contrast in laryngeal vestibule (0) & $145(82.39)$ & $58(81.69)$ \\
\hline & $\begin{array}{l}\text { Incomplete; narrow column air/contrast in laryngeal vesti- } \\
\text { bule (1) }\end{array}$ & $31(17.61)$ & $13(18.31)$ \\
\hline & None; wide column air/contrast in laryngeal vestibule (2) & $0(0)$ & $0(0)$ \\
\hline \multirow[t]{4}{*}{ \#14 Pharyngoesophageal segment opening } & $\begin{array}{l}\text { Complete distention and complete duration; no obstruction } \\
\text { of flow (0) }\end{array}$ & $63(35.80)$ & $27(38.03)$ \\
\hline & $\begin{array}{l}\text { Partial distention/partial duration; partial obstruction of flow } \\
\text { (1) }\end{array}$ & $113(64.20)$ & $41(57.74)$ \\
\hline & $\begin{array}{l}\text { Minimal distention/partial duration; marked obstruction of } \\
\text { flow (2) }\end{array}$ & $0(0)$ & $3(4.23)$ \\
\hline & No distention with total obstruction of flow (3) & $0(0)$ & $0(0)$ \\
\hline
\end{tabular}

are especially encouraging given that participants had minimal training (i.e., one practice swallow) prior to performing two effortful swallows during the videofluoroscopic evaluation. Having an inexpensive, non-invasive, portable, and easy-to-use method for providing biofeedback during dysphagia treatment would significantly improve current dysphagia management of patients by providing clinicians and patients with immediate insight into performance of swallowing maneuvers and exercises such as the effortful swallow. These findings expand upon previous research studies that have demonstrated the potential of HRCA as an effective dysphagia screening method and adjunct to VF when instrumental swallow evaluations are not feasible and provide evidence to support pursuing HRCA as a biofeedback method. Interestingly, in addition to these findings, we did not detect a statistically significant difference in MBSImP component scores (\#9, \#11, \#14) between non-effortful and effortful swallows. These results contribute to the mixed evidence base examining differences between non-effortful and effortful swallows in healthy adults [29, 30]. It is also possible that MBSImP ratings may not be sensitive enough to detect subtle changes in swallowing physiology because the MBSImP is a somewhat subjective, ordinal rating scale with a limited range of scores. This may particularly be true in the present study because all participants were healthy community dwelling adults with no report of swallowing difficulties; leading to a ceiling effect with MBSImP ratings.

Future studies should replicate this research work by examining HRCA's ability to classify non-effortful and effortful swallows with a larger sample of swallows that includes swallows from both healthy adults and patients with dysphagia. Including a larger and more variable sample of swallows will assist in improving the accuracy of the supervised machine classifier and may also allow us to detect differences in MBSImP component scores between non-effortful and effortful swallows. In addition to this, future studies should examine HRCA's ability to provide real-time continuous biofeedback during a treatment session targeting effortful swallows. It will be important to explore HRCA's ability to provide insight into performance of other swallowing maneuvers/exercises that would benefit from biofeedback (e.g., Mendelsohn maneuver) as well.

\section{Limitations}

The purpose of this research study was to determine the efficacy of HRCA as an inexpensive, non-invasive, and portable dysphagia biofeedback method. Because of the preliminary nature of this study, a relatively small sample of swallows were included for analyses $(n=247)$, which may have resulted in inadequate statistical power for comparing MBSImP component scores between non-effortful and effortful swallows. In addition to this, only swallows from healthy community dwelling adults across the lifespan were included in the analysis and only three MBSImP component scores were examined. This likely limited the range of swallows included (e.g., finite range of MBSImP component scores, limited severity range) and also limits the generalization of findings to patients with dysphagia in clinical settings. Due to time constraints while collecting data in a University hospital, participants received minimal training or practice prior to completing effortful swallows, which may have impacted their performance. In addition to this, we did not confirm accurate performance of effortful swallows with a validated measurement tool such as sEMG or manometry, 
so it is possible not all participants performed this compensatory maneuver correctly. Data were collected using a strict, standardized VF protocol to minimize radiation exposure to healthy community dwelling adults. As such, participants only swallowed thin liquid boluses and only two effortful swallows were collected from each participant during the VF procedure. Future studies should examine HRCA's ability to classify non-effortful and effortful swallows across various conditions (e.g., bolus volume, bolus viscosity, utensil) and across more trials from each participant.

\section{Conclusion}

This preliminary study found that HRCA signal features combined with decision trees and linear discriminant analysis classified swallows as non-effortful or as an effortful swallow with up to $76 \%$ accuracy, $76 \%$ sensitivity, and $77 \%$ specificity. These results provide promising evidence regarding the efficacy of using HRCA as a monitoring system and biofeedback method for dysphagia treatment in the future. Future studies should expand upon these findings to improve the machine learning algorithm performance and to further validate HRCA as a biofeedback method by analyzing a larger number of swallows (e.g., patient and healthy community dwelling adults) and by exploring the efficacy of using HRCA as a biofeedback method for other dysphagia treatment targets (e.g., Mendelsohn maneuver). This inexpensive, non-invasive, and portable method has the potential to transform dysphagia rehabilitation by providing real-time feedback regarding treatment performance.

Acknowledgements People: Thanks are due to Brynn Jones-Rastelli, M.S. CCC-SLP, Emma Becker, B.A., Amanda S. Mahoney, M.A. SLP, and Erin Lucatorto M.A. CCC-SLP for assistance with data collection and coding.

Funding Research reported in this publication was supported by the Eunice Kennedy Shriver National Institute of Child Health \& Human Development of the National Institutes of Health under Award Number R01HD092239, while the data were collected under Award Number R01HD074819. The content is solely the responsibility of the authors and does not necessarily represent the official views of the National Institutes of Health.

\section{Declarations}

Conflict of interest We have no conflict of interest to declare.

Ethical Approval All procedures performed in studies involving human participants were in accordance with the ethical standards of the institutional and/or national research committee and with the 1964 Helsinki declaration and its later amendments or comparable ethical standards.

Informed Consent Informed consent was obtained from all individual participants included in the study.

\section{References}

1. Crary MA, Groher ME. Basic concepts of surface electromyographic biofeedback in the treatment of dysphagia: a tutorial. Am J Speech Lang Pathol. 2000;9:116. https://doi.org/10.1044/10580360.0902.116

2. Leder SB, Novella S, Patwa H. Use of fiberoptic endoscopic evaluation of swallowing (FEES) in patients with amyotrophic lateral sclerosis. Dysphagia. 2004;19:177-81. https://doi.org/10.1007/ s00455-004-0009-2.

3. Azola AM, Sunday KL, Humbert IA. Kinematic visual biofeedback improves accuracy of learning a swallowing maneuver and accuracy of clinician cues during training. Dysphagia. 2017;32:115-22. https://doi.org/10.1007/s00455-016-9749-z.

4. Vose AK, Marcus A, Humbert I. Kinematic visual biofeedback improves accuracy of swallowing maneuver training and accuracy of clinician cues during training in stroke patients with dysphagia. PM R. 2019;11:1159-69. https://doi.org/10.1002/pmrj.12093.

5. Azola AM, Greene LR, Taylor-Kamara I, Macrae P, Anderson C, Humbert IA. The relationship between submental surface electromyography and hyo-laryngeal kinematic measures of Mendelsohn maneuver duration. J Speech Lang Hear Res. 2015;58:1627-36. https://doi.org/10.1044/2015_JSLHR-S-14-0203.

6. Benfield JK, Everton LF, Bath PM, England TJ. Does therapy with biofeedback improve swallowing in adults with dysphagia? A systematic review and meta-analysis. Arch Phys Med Rehabil. 2019;100:551-61. https://doi.org/10.1016/j.apmr.2018.04.031.

7. Reddy NP, Simcox DL, Gupta V, Motta GE, Coppenger J, Das A, et al. Biofeedback therapy using accelerometry for treating dysphagic patients with poor laryngeal elevation: case studies. J Rehabil Res Dev. 2000;37:361-72.

8. Li C-M, Wang T-G, Lee H-Y, Wang H-P, Hsieh S-H, Chou M, et al. Swallowing training combined with game-based biofeedback in poststroke dysphagia. PM R. 2016;8:773-9. https://doi.org/10. 1016/j.pmrj.2016.01.003.

9. Li C-M, Lee H-Y, Hsieh S-H, Wang T-G, Wang H-P, Chen J-JJ. Development of innovative feedback device for swallowing therapy. J Med Biol Eng. 2016;36:357-68. https://doi.org/10.1007/ s40846-016-0146-8.

10. Kantarcigil C, Kim MK, Chang T, Craig BA, Smith A, Lee CH, et al. Validation of a novel wearable electromyography patch for monitoring submental muscle activity during swallowing: a randomized crossover trial. J Speech Lang Hear Res. 2020;63:3293310. https://doi.org/10.1044/2020_JSLHR-20-00171.

11. Dudik JM, Coyle JL, Sejdić E. Dysphagia screening: contributions of cervical auscultation signals and modern signal-processing techniques. IEEE Trans Hum Mach Syst. 2015;45:465-77. https:// doi.org/10.1109/THMS.2015.2408615.

12. Sejdić E, Steele CM, Chau T. Classification of penetration-aspiration versus healthy swallows using dual-axis swallowing accelerometry signals in dysphagic subjects. IEEE Trans Biomed Eng. 2013;60:1859-66. https://doi.org/10.1109/TBME.2013.2243730.

13. Dudik JM, Jestrović I, Luan B, Coyle JL, Sejdić E. A comparative analysis of swallowing accelerometry and sounds during saliva swallows. Biomed Eng Online. 2015;14:3. https://doi.org/10. 1186/1475-925X-14-3.

14. Dudik JM, Kurosu A, Coyle JL, Sejdić E. A comparative analysis of DBSCAN, K-means, and quadratic variation algorithms for automatic identification of swallows from swallowing accelerometry signals. Comput Biol Med. 2015;59:10-8. https://doi.org/10. 1016/j.compbiomed.2015.01.007.

15. Jestrović I, Dudik JM, Luan B, Coyle JL, Sejdić E. Baseline characteristics of cervical auscultation signals during various head maneuvers. Comput Biol Med. 2013;43:2014-20. https://doi.org/ 10.1016/j.compbiomed.2013.10.005. 
16. Dudik JM, Coyle JL, El-Jaroudi A, Mao Z-H, Sun M, Sejdić E. Deep learning for classification of normal swallows in adults. Neurocomputing. 2018;285:1-9. https://doi.org/10.1016/j.neucom.2017.12.059.

17. Yu C, Khalifa Y, Sejdic E, Silent Aspiration Detection in High Resolution Cervical Auscultations, IEEE EMBS International Conference on Biomedical \& Health Informatics (BHI), 2019.

18. Mao S, Zhang Z, Khalifa Y, Donohue C, Coyle JL, Sejdic E. Neck sensor-supported hyoid bone movement tracking during swallowing. R Soc Open Sci. 2019;6:181982. https://doi.org/10.1098/rsos. 181982.

19. Donohue C, Mao S, Sejdić E, Coyle JL. Tracking hyoid bone displacement during swallowing without videofluoroscopy using machine learning of vibratory signals. Dysphagia. 2020. https:// doi.org/10.1007/s00455-020-10124-Z.

20. Khalifa Y, Donohue C, Coyle JL, Sejdic E. Upper esophageal sphincter opening segmentation with convolutional recurrent neural networks in high resolution cervical auscultation. IEEE J Biomed Health Inform. 2021;25:493-503. https://doi.org/10.1109/ JBHI.2020.3000057.

21. Donohue C, Khalifa Y, Perera S, Sejdić E, Coyle JL. How closely do machine ratings of duration of UES opening during videofluoroscopy approximate clinician ratings using temporal kinematic analyses and the mbsimp? Dysphagia. 2020. https://doi.org/10. 1007/s00455-020-10191-2.

22. Mao S, Sabry A, Khalifa Y, Coyle JL, Sejdic E. Estimation of laryngeal closure duration during swallowing without invasive X-rays. Future Gener Comput Syst. 2021;115:610-8. https://doi. org/10.1016/j.future.2020.09.040.

23. Kurosu A, Coyle JL, Dudik JM, Sejdic E. Detection of swallow kinematic events from acoustic high-resolution cervical auscultation signals in patients with stroke. Arch Phys Med Rehabil. 2019;100:501-8. https://doi.org/10.1016/j.apmr.2018.05.038.

24. Donohue C, Khalifa Y, Perera S, Sejdić E, Coyle JL. A preliminary investigation of whether HRCA signals can differentiate between swallows from healthy people and swallows from people with neurodegenerative diseases. Dysphagia. 2020. https://doi.org/ 10.1007/s00455-020-10177-0.

25. Martin-Harris B, Brodsky MB, Michel Y, Castell DO, Schleicher M, Sandidge J, et al. MBS measurement tool for swallow impairment-MBSImp: establishing a standard. Dysphagia. 2008;23:392-405. https://doi.org/10.1007/s00455-008-9185-9.

26. Bülow M, Olsson R, Ekberg O. Videomanometric analysis of supraglottic swallow, effortful swallow, and chin tuck in patients with pharyngeal dysfunction. Dysphagia. 2001;16:190-5. https:// doi.org/10.1007/s00455-001-0065-9.
27. Bülow M, Olsson R, Ekberg O. Supraglottic swallow, effortful swallow, and chin tuck did not alter hypopharyngeal intrabolus pressure in patients with pharyngeal dysfunction. Dysphagia. 2002;17:197-201. https://doi.org/10.1007/s00455-002-0050-y.

28. Felix VN, Corrêa SMA, Soares RJ. A therapeutic maneuver for oropharyngeal dysphagia in patients with Parkinson's disease. Clinics. 2008;63:661-6. https://doi.org/10.1590/s1807-59322 008000500015.

29. Bahia MM, Lowell SY. A systematic review of the physiological effects of the effortful swallow maneuver in adults with normal and disordered swallowing. Am J Speech Lang Pathol. 2020;29:165573. https://doi.org/10.1044/2020_AJSLP-19-00132.

30. Molfenter SM, Hsu C-Y, Lu Y, Lazarus CL. Alterations to swallowing physiology as the result of effortful swallowing in healthy seniors. Dysphagia. 2018;33:380-8. https://doi.org/10.1007/ s00455-017-9863-6.

31. Takahashi K, Groher ME, Michi K. Methodology for detecting swallowing sounds. Dysphagia. 1994;9:54-62. https://doi.org/10. 1007/BF00262760.

32. Shrout PE, Fleiss JL. Intraclass correlations: uses in assessing rater reliability. Psychol Bull. 2005;86:420.

33. Khalifa Y, Coyle JL, Sejdić E. Non-invasive identification of swallows via deep learning in high resolution cervical auscultation recordings. Sci Rep. 2020;10:8704. https://doi.org/10.1038/ s41598-020-65492-1.

34. Dudik JM, Jestrovic I, Luan B, Coyle JL, Sejdic E. Characteristics of dry chin-tuck swallowing vibrations and sounds. IEEE Trans Biomed Eng. 2015;62:2456-64. https://doi.org/10.1109/TBME. 2015.2431999.

Publisher's Note Springer Nature remains neutral with regard to jurisdictional claims in published maps and institutional affiliations.

\section{Cara Donohue PhD CCC-SLP}

\section{Yassin Khalifa MS}

Subashan Perera $\mathrm{PhD}$

Ervin Sejdić $\mathrm{PhD}$

James L. Coyle PhD, CCC-SLP, BCS-S 